\title{
Linx
}

Revue des linguistes de l'université Paris X Nanterre

56 | 2007

Linguistique des genres

\section{Contribution à une linguistique néo-saussurienne des genres de la parole (2) : analyse des valeurs d'indexicalité interlocutoire de on selon les genres textuels}

Denise Malrieu

\section{CpenEdition}

Journals

Édition électronique

URL : http://journals.openedition.org/linx/377

DOI : $10.4000 / \operatorname{lin} \times .377$

ISSN : 2118-9692

Éditeur

Presses universitaires de Paris Nanterre

Édition imprimée

Date de publication : 1 juin 2007

Pagination : 157-178

ISSN : 0246-8743

Référence électronique

Denise Malrieu, « Contribution à une linguistique néo-saussurienne des genres de la parole (2) : analyse des valeurs d'indexicalité interlocutoire de on selon les genres textuels », Linx [En ligne], 56 | 2007, mis en ligne le 21 février 2011, consulté le 19 avril 2019. URL : http://

journals.openedition.org/linx/377 ; DOI : 10.4000/linx.377 


\title{
Contribution à une linguistique néo-saussurienne des genres de la parole (2) : analyse des valeurs d'indexicalité interlocutoire de on selon les genres textuels
}

\author{
Denise Malrieu, UMR MODYCO, Paris $X$ \\ dmalrien@u-paris10.fr
}

\section{Les objectifs}

Je reprendrai les propositions de S. Bouquet (Langages $n^{\circ} 153$ ) concernant la possibilité de faire se rejoindre linguistique de la langue et linguistique de la parole : la linguistique de la langue dans ses grammaires ne décrit que des parties détachées de sens, la linguistique de la parole va définir une pragmatique du sens qui a trait au genre et à la façon dont des traits suprasegmentaux du texte global et de ses sous-ensembles, vont contraindre l'analyse grammaticale du segmental. Le constat de la très grande diversité des usages du on selon les domaines et genres de textes m'a amenée à faire des hypothèses sur le réglage de cet usage par les genres.

La démarche dessinée ici voudrait préciser les thèses de Bakhtine sur la normativité des genres. «Le vouloir dire du locuteur se réalise avant tout dans le choix d'un genre de discours. Nous disposons d'un riche répertoire de genres de discours oraux (et écrits). Ce choix se détermine en fonction de la spécificité d'une sphère donnée de l'échange verbal, des besoins d'une thématique, de l'ensemble constitué des partenaires. Pour parler nous nous servons toujours des genres du discours, autrement 
dit, tous nos énoncés disposent d'une forme type et relativement stable de structuration d'un tout. Si les genres du discours n'existaient pas, et si nous n'en avions pas la maitrise, et qu'il nous faille les créer pour la première fois dans le processus de la parole, qu'il nous faille construire chacun de nos énoncés, l'échange verbal serait quasiment impossible.

Le locuteur reçoit outre les formes prescriptives de la langue commune, les formes non moins prescriptives pour lui de l'énoncé, c'est-à-dire des genres du discours - pour une intelligence entre locuteurs ces derniers sont aussi indispensables que les formes de langue.»

On peut reformuler comme suit les différentes composantes du genre dessinées par Bakhtine :

i) les modalités physiques de la communication: écrit, oral, support de la communication, contraintes de la situation d'énonciation marquant entre autres les modalités interlocutives.

ii) Socialité et structuration sociale de l'échange : normativité des faces et des modes d'adresse, registres de langue plus ou moins soutenus.

iii) le domaine d'activité et la thématique de l'échange : normativité des domaines : type de rapport à la langue et types de discours : mode de référenciation et désignation, de cohérence discursive, de construction des actants, d'argumentation, etc ${ }^{1}$.

iv) le genre textuel (oral ou écrit) en tant que «forme type et relativement stable de structuration d'un tout», mettant en jeu de façon réglée différentes sémiotiques dans un champ donné de pratique: le genre condense les instructions liées au contrat énonciatif (roman vs autobiographie); la structuration du signifiant au niveau global, indexe la fonction discursive d'une partie par rapport au tout, et donne des instructions interprétatives propres aux parties.

La notion de genre doit apporter des informations sur les conditions de possibilité de types d'énoncés à l'intérieur de la normativité des pratiques culturelles, c'est-à-dire apporter des éléments pour départager l'acceptabilité de tel énoncé, que le système de la langue ne fournit pas, éléments qui renvoient à une énonciation centrée non pas sur le sujet mais sur les formes de dialogalité informées par le genre et aux dimensions réflexives de la parole. En prenant comme objet les variations de l'usage du on selon les genres, je ne réduis pas la question à la référenciation mais l'objet est plutôt de définir des familles de types d'énoncés où on a été choisi pour référer. Sans forcément adopter le point de vue kantien des schèmes a priori soutenu par Forest (2003), on peut envisager l'énoncé comme un type ou signe linguistique. La problématique n'est pas seulement de définir en quoi le genre appellerait l'usage privilégié de certains types mais aussi de repérer les discordances ou tensions entre le système de la langue et les conditions de sa mise en œuvre dans l'énonciatif. La normativité du genre comme source de la variation.

\footnotetext{
${ }^{1}$ Même s'il est commode d'opposer de ce point de vue discours politique, scientifique, poétique par ex, il ne me parait guère possible de parler de «discours de domaines", car, à l'intérieur d'un domaine, les genres sont très hétérogènes et ce sont eux qui peuvent donner lieu à analyse contrastive.
} 


\section{La démarche}

Dans son article programmatique sur la typologie et la caractérisation des genres littéraires, Schaeffer (2006) nous dit : « Nous devons mettre les caractéristiques identifiées par les noms [de genre] en rapport avec une analyse indépendante des textes identifiés par ces noms (...). Il est important en effet que l'analyse textuelle soit menée par des moyens indépendants. Ceci implique que nous devons disposer d'une méthode ou mieux d'un ensemble de méthodes, mises au point pour étudier les discours et les textes, indépendamment de la problématique générique. Il nous faut en somme nous engager dans une analyse stratifiée des textes, commençant au niveau linguistique et finissant au niveau des macrostructures discursives, par exemple de type narratologique ou thématique. Il nous faut aussi prendre en compte la dimension temporelle de la série des textes identifiés par un même nom. »

S. Bouquet, de son côté, pose que la linguistique du genre revient à opposer deux énoncés homonymes différenciables par au moins un trait grammatical induit par le genre.

Les deux programmes sont différents: celui de Schaeffer serait d'établir une caractérisation linguistique et discursive des genres par approche quantitative sur corpus, celle de Bouquet serait d'identifier quels sont les traits de genre qui contraignent l'interprétation sémantique (via la grammaire et la syntaxe) de l'énoncé. La proposition de S. Bouquet, si elle est justifiée dans son exigence de rester sur le terrain de la linguistique, présente l'avantage de ne reposer sur aucune typologie a priori des genres; si elle parait praticable pour des contextes oraux restreints, elle pose des problèmes pour les énoncés attestés dans les textes sur lesquels il est difficile de projeter fictivement un genre et présente des limites, celles de l'imagination du linguiste concernant l'existence d'une ambiguité sémantique sur des énoncés isolés. De plus, la linguistique des exemples (vs de corpus) a du mal à prendre en compte la complexité sémiotique des genres textuels, leur complexité structurelle et effets à longue distance, du fait de la tradition logico-grammaticale restreignant la linguistique à la linguistique de la phrase. Est-il possible d'établir un pont entre les deux démarches ? Je dirai qu'une passerelle est lancée.

Le programme de Schaeffer pose plusieurs questions : qu'entend-il par «il est important que l'analyse textuelle soit menée par des moyens indépendants»? Préconise-t-il l'emploi des méthodes objectivantes telles l'analyse factorielle de traits morpho-syntaxiques ou lexicaux? La démarche proposée ici diffère de l'analyse quantitative lexicométrique dans la mesure où l'analyse factorielle classique travaille sur les mots isolés et ne peut caractériser la sémantique de ces unités qu'en réinjectant dans les graphiques d'analyse factorielle une sémantique lexicale décontextualisée donc doxique, en risquant d'attribuer une cohérence sémantique a posteriori aux cooccurrences observées, lieu des interprétations compulsives éminemment incontrôlées. L'articulation d'une sémantique interprétative et de la linguistique de corpus n'est pas encore élaborée, cette dernière devant déplier, désintriquer les dimensions hétérogènes de la parole, implique, pour être efficace, une théorie des genres : celle-ci exige d'élaborer une pragmatique expérimentale, i.e. de travailler au niveau de l'énoncé (dans ses propriétés grammaticales, syntaxiques, prosodiques) plongé dans un contexte dont on fait varier les propriétés. On serait ainsi à l'opposé de l'analyse indépendante des genres 
et des propriétés textuelles que propose Schaeffer, puisque la définition des genres serait relative aux nécessités de l'interprétation locale ; et réciproquement, la finesse de l'analyse grammaticale dépendra de la richesse et de la diversité du corpus analysé. À la différence des démarches purement quantitatives, la démarche adoptée ici s'adosse à la compétence interprétative du lecteur pour rendre compte de l'interprétation par l'analyse différentielle des traits segmentaux et suprasegmentaux.

En conséquence, ma démarche diverge aussi de la proposition de Schaeffer quant à la chronologie des analyses, puisque, comme on le verra, c'est la méthodologie qui conjoint les différents niveaux d'analyse textuelle (en l'occurrence la méthodologie $\mathrm{XML}$ ) qui autorise l'analyse différentielle de tous types de traits portés par une unité du texte. Ce type de modélisation empirique, suppose évidemment des hypothèses sur les traits de genres pertinents comme une modélisation des traits grammaticaux différentiels du morphème. L'idée retenue est donc de travailler sur des textes attestés et de définir un corpus comme un ensemble de données expérimentales dont on va faire jouer de façon différentielle certains traits de genres pour l'analyse comparative des traits grammaticaux du morphème.

Une première définition a priori des genres textuels tient compte à la fois des domaines d'activité (littérature vs journalisme), des champs génériques (genres narratifs vs théâtre vs poésie), des genres (dans les genres narratifs, on distinguera le conte, le roman, la biographie, etc.) (Malrieu \& Rastier, 2001). Mais une définition plus précise doit parvenir à articuler les 4 facteurs cités plus haut de façon systématique et différentielle.

En tant que pronom personnel et pronom indéfini, le on touche à un grand nombre de domaines de l'énonciation dans la langue :

- dans le rapport de l'énonciateur à autrui, le on concerne a) l'interlocutivité et l'attribution des discours aux différents participants potentiels de la scène énonciative (indexicalité interlocutoire) ; b) il concerne aussi la socialité, liée aux rôles sociaux et aux normes d'adresse selon les contextes d'interlocution ainsi que les registres de parole plus ou moins soutenus ; c) l'intersubjectivité et l'échangeabilité des points de vue, mais aussi réflexivité du locuteur; d) les phénomènes d'assomption ou d'effacement du locuteur.

- dans le rapport de l'énonciateur au monde dicible, le on touche à la référentialité, l'indexicalité intratextuelle, et l'actualisation dans la construction d'un univers partagé : déixis, anaphore et définitude.

- Dans l'actanciation, le on portant le trait +Humain, et le trait argumental +Sujet, va interagir avec la voix, ergativité et patientivité, en particulier dans le discours sur ego et dans les énoncés où ego est objet direct ou indirect du verbe dont on est le sujet.

Notre analyse se centrera sur les deux premiers aspects: les rapports d'interlocution et la référentialité : la grammaire différentielle de on proposée par S. Bouquet est relative à l'indexicalité des pronoms personnels. Ne sont pas envisagés les formes syntaxiques vicariantes du on non sujet, ni les phénomènes liés à l'actanciation, $\mathrm{ni}$ les formes équivalentes du on (certains impersonnels et formes infinitives ou passives par ex). Dans la démarche dessinée ici, l'analyse différentielle peut prendre deux formes : analyse quantitative des répartitions de valeurs selon les genres, i.e. des corrélations entre traits de genres et traits grammaticaux, analyse qualitative des 
processus interprétatifs induits par le genre (cf. 3.2 et 3.3). La première tâche qui m'incombe ici est donc de définir les traits de genres.

\subsection{Le corpus et les genres}

2.1.1. Le corpus : il se compose de 6 romans dont 5 de M. Duras (Un Barrage contre le Pacifique, Moderato cantabile, Dix heures et demi du soir en été, L'Amant de la Chine du Nord (ACN), Le Ravissement de Lol V. Stein, un roman de Stendhal, La Charteuse de Parme; un ouvrage d'entretiens entre MD et une journaliste, série d'entretiens retranscrits de façon fidèle, Les Parleuses; La vie matérielle est une série d'entretiens (propos retranscrits entre MD et Jérôme Beaujour, retravaillés par l'auteur, en cela il se distingue des Parlenses: il s'agit d'une écriture d'auteur); enfin deux ouvrages recueils d'articles de presse grand public écrits sur plusieurs années par MD : Outside et Le monde extérieur.

\begin{tabular}{|l|c|r|}
\hline Genre & Nb d'ouvrages & Nb de mots \\
\hline romans & 6 & 399366 \\
\hline Entretiens (bâtons rompus) Parleuses & 1 & 68822 \\
\hline Entretiens retravaillés & 1 & 35345 \\
\hline Recueils d'articles de presse & 2 & 140119 \\
\hline
\end{tabular}

Tableau 1 : Composition du corpus

Le sous-corpus d'articles de presse se compose de 16 récits hétérodiégétiques, 29 récits autobiographiques, 13 interviews, 26 commentaires d'oeuvre, 22 commentaires d'actualité, 20 articles de réflexions générales.

Les traits pris en compte dans la constitution du corpus peuvent être décrits dans l'arborescence suivante :

- fiction

+oral retranscrit (entretiens)

+ interview dissymétrique (-familiarité)

discours descriptif sur ego : Outside_entretiens

- interview dissymétrique

+Discussion à bâtons rompus (+familiarité) : Les parleuses

-Discussion à bâtons rompus (propos recueillis)

-oral retranscrit (articles)

+ récit

- discours rapportés (discours du narrateur)

+ homodiégétique. (autobiographique) :士PRES : Vie matérielle,

Outside, Monde extérieur

- homodiégétique : Outside_récit

+ discours rapportés (DD, DI, etc.) - récit

+ réflexions générales : Vie matérielle_réflexions

- réflexions générales : Monde extérieur_commentaire d'actualité

+ fiction (roman) 


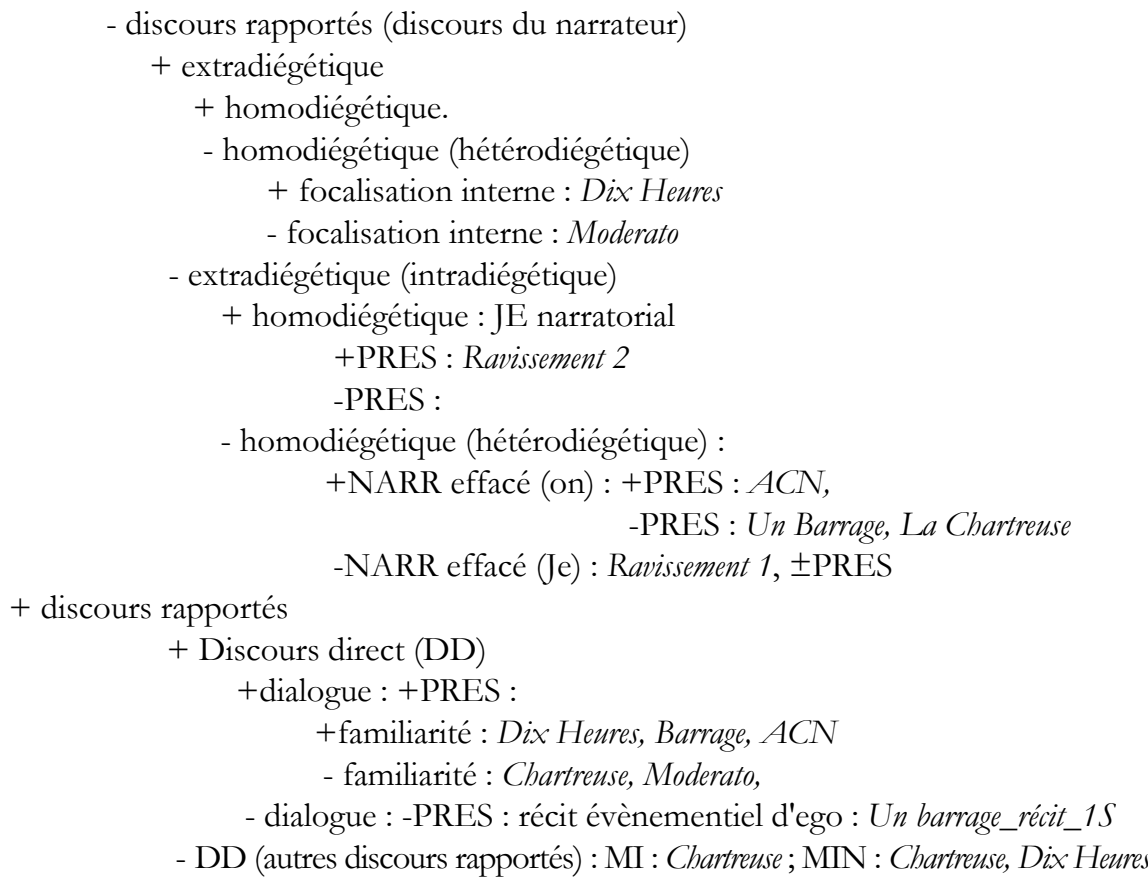

On peut, à un premier niveau, opposer les genres fictionnels aux genres non fictionnels. Dans les genres non fictionnels les interlocuteurs sont explicitement définis par la situation interlocutive, alors que les genres fictionnels changent le centre déictique par mimésis de l'interlocution non fictionnelle.

On peut opposer dans les genres non fictionnels, les genres oraux retranscrits (interview, entretien discussion) aux genres écrits (récits; réflexions générales, commentaires d'actualité) sur le trait de l'interlocutivité directe vs médiée.

Examinons tout d'abord les genres oraux non fictionnels représentés dans le corpus :

- En ce qui concerne les entretiens (échange dual en face à face) : on peut distinguer les entretiens informels (discussion à bâtons rompus où les rôles sont quasi symétriques) vs interview dissymétrique où les rôles sont bien distincts et plus formels vs propos recueillis. L'indexicalité extratextuelle interlocutoire du locuteur amplifiée à l'allocutaire sera quasi absente chez l'interviewé, plus présente dans la discussion à bâtons rompus, quasi absente dans les propos recueillis. Dans une conférence publique, échange en face à face formel réglé, le on peut désigner l'allocutaire pluriel comme le locuteur, de même dans un roman, mais pas le je amplifié à l'allocutaire singulier.

- Dans le récit évènementiel d'ego en face à face, selon que les relations interlocutoires sont entachées de familiarité ou pas, le on désignant le locuteur amplifié sera admis ou non à la place du nous.

- Dans l'entretien asymétrique, selon la thématique de l'entretien, l'interviewé peut avoir un discours sur "le monde », objets non interlocutifs ou au contraire sur un ego délocuté. Dans ce dernier cas, l'activité discursive peut être de type narratif ou de description de soi en tant que représentant d'une classe ou en tant 
que membre d'un groupe social. Je renvoie à la partie 4 pour une ébauche des traits distinctifs des deux types de discours (discours sur ego membre de la classe vs du groupe).

- Dans les genres écrits non fictionnels, la présence du locuteur et l'adresse au lecteur peuvent être marquées ou effacées. Les récits d'articles de presse sont généralement des documentaires à visée proche de la fable, l'auteur est en relation interlocutive plus directe avec son lecteur que dans le récit fictionnel et peut se permettre de faire union avec lui pour emporter son adhésion.

Dans les écrits narratifs fictionnels, le cadre change.

Ainsi, dans le roman, les rapports énonciatifs du narrateur sont par définition marqués par :

- la place du narrateur (présente ou effacée) : en accord avec Genette (1983) on peut affirmer des degrés variables de présence du narrateur.

Le trait intradiégéticité entraîne la présence d'une indexicalité extratextuelle interlocutoire : dans le discours du narrateur primaire, le jet désigne le narrateur, qui peut commenter son activité narrative, et/ou s'adresser au lecteur de façon explicite. Le choix du on plutôt que du je permet l'effacement énonciatif du narrateur, ou plutôt que du $t u$, l'adresse indirecte au narrataire (voir La Chartreuse). À la différence du récit oral, le on inclusif (du narrateur amplifié au narrataire) n'est guère envisageable. Le trait extradiégéticité exclut cette indexicalité dans le discours du narrateur. Le narrateur, comme le souligne Genette, est toutefois présent dans le récit, qu'il soit extra- ou intradiégétique, soit dans la construction de la scène et du point de vue : on sujet de verbes de perception (Rabatel, 1998) soit dans les modalisations épistémiques qui ponctuent cette donation ${ }^{2}$. Ce on, constructeur de point de vue et de focalisation du regard, est, comme dans le cinéma (cf. l' $A C N$ ) la façon d'englober le narrataire dans le on (Maingueneau, 2000).

Il faut ensuite examiner la combinatoire des traits extra-/intradiégéticité et homo-/hétérodiégéticité ${ }^{3}$ : - comme dans toute interlocution mimétique, dans l'extrabomodiégéticité, le pronom je1 du narrateur est effacé au profit du jez du personnage, sorte de désignateur rigide qui n'est défini que par les prédications internes au texte et ce je n'a pas d'allocutaire, même s'il convoque implicitement une interlocutivité (impliquée par la 1S) avec le narrataire ; le je reprend sa valeur d'indexicalité interlocutoire dans les situations interlocutives représentées de l'univers fictionnel. Le on désignant le narrateur ne peut exister que dans les activités narratives de construction de la scène (" on voyait») ou dans les modalisations épistémiques (" on dirait, on eût dit»). Le on désignant le personnage correspond à un je amplifié par anaphore. On notera que dans le récit intra- homodiégétique, le je1 du narrateur ne peut être remplacé par le on, comme c'est le cas pour le récit intra- hétérodiégétique.

\footnotetext{
${ }^{2}$ La présence du narrateur s'exprime aussi bien dans les choix entre discours narrativisé ou mimésis des différents discours rapportés.

${ }^{3}$ Nous n'envisageons pas ici toutes les configurations possibles de la personne narratoriale comme le fait Fludernick, (1996), en particulier le récit à la deuxième personne, qui favorise l'identification du lecteur.
} 


\begin{tabular}{|c|c|c|}
\hline $\begin{array}{l}J e \text { dans le discours du } \\
\text { narrateur }\end{array}$ & Récit homodiégétique & Récit hétérodiégétique \\
\hline $\begin{array}{l}\text { Récit intradiégétique } \\
\text { (en je ou en on) }\end{array}$ & $\begin{array}{l}+\mathrm{IDX}+\mathrm{EXT}+\mathrm{ITL}: j e_{1} \text { du } \\
\text { narrateur (on du narrataire, } \\
\text { non du narrateur sauf par } \\
\text { modalisation épistémique) } \\
+\mathrm{IDX}+\mathrm{EXT}-\mathrm{ITL}=j e_{2} \text { du } \\
\text { personnage (on }=j e_{2} \text { du person- } \\
\text { nage amplifié par anaphore, } \\
\text { ou par construction de la scène) }\end{array}$ & $\begin{array}{l}+\mathrm{IDX}+\mathrm{EXT}+\mathrm{ITL} \text { (on=je } e_{1} \\
\text { du narrateur et } t u \text { du narrataire } \\
\text { par effacement énonciatif) } \\
\text { (La Chartreuse) }\end{array}$ \\
\hline Récit extradiégétique & $\begin{array}{l}+\mathrm{IDX}+\mathrm{EXT}-\mathrm{ITL}=j e_{2} \mathrm{du} \\
\text { personnage (on }=j e_{2} \text { du person- } \\
\text { nage amplifié par anaphore, } \\
\text { ou on par construction de la } \\
\text { scène avec focalisation interne } \\
\text { ou par modalisation) }\end{array}$ & $\begin{array}{l}\text { Construction de la scène (« on } \\
\text { voyait ») } \\
\text { Modalisation épistémique } \\
\text { (« on eût dit») }\end{array}$ \\
\hline
\end{tabular}

Tableau $2:$ valeurs indexicales extratextuelles $d u$ on narratorial

On notera aussi que le je homodiégétique, du fait de son statut non interlocutoire délocutif se voit rapproché de la troisième personne, ce qui rend compréhensible le passage de l'un à l'autre dans certaines œuvres, (voir dans l'Amant) voir aussi la 3S, dans le monologue intérieur narrativisé). On voit là les contraintes liées au genre: la prise de distance que représente le passage à la $3 \mathrm{~S}$ dans le récit autobiographique romanesque ne sera pas autorisée dans le récit autobiographique.

- enfin, le rapport narrateur/narrataire est intrinsèquement marqué par la dissymétrie des savoirs et les jeux épistémiques du narrateur. L'omniscience du narrateur hétérodiégétique peut être assumée ou niée : l'omniscience niée (très marquée chez un auteur comme M. Duras) va se traduire par des modalités narratives de la nontransparence (dans la dénomination retardée ou absente, les modalisations épistémiques du doute et du non savoir, les référenciations indéfinies). L'intra- et hétérodiégéticité favorisent la déclaration de non-omniscience du narrateur et toutes les modalisations épistémiques concernant la construction d'univers ${ }^{4}$ où le on sujet de modaux est fréquent (on dirait, etc.).

\subsubsection{Le balisage $d u$ corpus}

Je ne m'étendrai pas sur cet aspect (cf. l'auteur, 2007) : l'idée centrale est de travailler sur des textes intégraux en sauvegardant leur structure, d'attribuer par le balisage XML, en fonction des besoins de l'analyse, tout trait nécessaire à la description de toute unité textuelle, quelle qu'en soit la longueur. Le balisage adopté

\footnotetext{
${ }^{4}$ Cf l'auteur : «Type de narrateur et place du lecteur dans Le ravissement de Lol V. Stein », où je montre que la configuration intra- et hétérodiégéticité de la première partie du roman permet la meilleure interaction problématisante pour le lecteur. (www.modyco.fr)
} 
concerne d'une part l'enrichissement du header ${ }^{5}$ proposé par la TEI pour ce qui concerne le <textClass $>$ : domaine, champ générique, genre et sous-genres définis plus haut, le balisage de la structure logique du texte est celui de la TEI; dans les séquences textuelles, le balisage des discours rapportés pour l'analyse de on s'imposait, j'ai introduit des balises de types de discours rapportés, leurs segments introducteurs et incises. Cela permet de situer chaque occurrence de on à l'intérieur des univers de discours représentés (DR ou discours du narrateur) et de la caractériser par l'ensemble des traits hérités du texte et de ses sous-ensembles.

La liste des discours rapportés balisés est la suivante : le discours direct (DD) sous ses différentes formes; le discours indirect, (DI), soit simple soit narrativisé (DIN) ; le monologue intérieur soit simple au DD (MI), au DI (MII), ou narrativisé (MIN souvent désigné DIL) ; le discours rapporté narrativisé (DRN souvent désigné DIL : «Il n'était pas bien, disait-il, et préférait rentrer»).

\section{3 - Propriétés des énoncés et grammaire du on}

\subsection{Situations interlocutives, normes de socialité et usage de on}

Concernant la socialité on peut distinguer ce qui relève de la norme dans un contexte culturel donné et ce qui relève du degré de proximité sociale et affective des interlocuteurs dans ce contexte, que je désignerai par familiarité. La norme interlocutive en français varie selon que l'on est dans un échange oral informel (conversation) ou formel (conférence) : dans les situations d'interlocution en face à face informelle interindividuelle, la norme est d'utiliser les pronoms interlocutoires. L'adresse à autrui par un pronom non interlocutoire (il ou on) établit une hiérarchie interlocutive avec traits d'ironie ou d'agressivité envers l'allocutaire. Dans l'échange en face à face formel, (conférence) le on peut s'adresser sans problème à l'allocutaire collectif. De même dans un écrit (article, roman) où l'allocutaire est un individu anonyme potentiellement collectif.

Le facteur de proximité sociale et affective joue dans le registre de langue, en particulier dans le remplacement du nous par le on, le nous devenant hypercorrect. Cet usage familier du on favorise une annexion qui ne se dit pas, de l'allocutaire.

Dans sa description des valeurs en discours de on, Moignet (1965, p.154 et sq) cite des exemples de remplacement de chacune des personnes du paradigme par le pronom on. Ces exemples sont tous tirés du théâtre classique du XVII' siècle. Il n'est pas évident que cette omnivalence du on soit encore présente dans le français contemporain.

\subsection{Interlocutivité et indexicalité interlocutoire des pronoms personnels}

Dans cette partie nous envisageons les normes d'usage du on selon les situations d'interlocution et la façon dont on se situe par rapport aux pronoms de l'interlocution.

Certains ont soutenu la thèse que on était un nominal et non un pronom car il ne peut être anaphorique (Grévisse \$461b), Rey-Debove (2001). Selon Moignet (1965),

${ }^{5}$ L'en-tête concerne toutes informations bibliographiques ou autres portant sur le texte dans sa globalité. 
«le on ne s'intègre proprement à aucun des sous-systèmes des personnels purs existentiels simples ou doubles (nous, vous) ou anaphoriques (il). Il tend à remplir la case vide de $3 \mathrm{~S}$ indéterminée et il se situe aussi au-delà de $i l s$, sans être un pronom anaphorique. p. 28 : le on signifie la personne sujet animée indéterminée... Tout en étant rattaché à la troisième personne, .... il peut signifier n'importe quelle personne, il ne discrimine pas le masculin du féminin, et convient à l'expression des deux nombres... Sous une forme de singulier il s'apparente psychiquement au pluriel. »

Je m'appuierai pour l'analyse sur la grammaire des pronoms personnels proposée par S. Bouquet (article ci-joint ${ }^{6}$ ) et sur la révision par A. Joly (1987) du système de la personne de Benveniste.

En accord avec la critique qu'adressent A. Joly ou G. Moignet à Benveniste (qui fait à tort de la $3^{\text {ème }}$ personne le pronom de «non-personne »), on distingue les personnes de l'interlocution (1 et 2) et les personnes hors interlocution (3). À leur suite, A. Joly distingue les pronoms prédicatifs, ontiques, sur le plan nominal de l'espace qui fonctionnent comme des noms (en particulier ils peuvent suivre une préposition ou le présentatif «c'est ») et les pronoms non prédicatifs, sur le plan verbal du temps, qui ne possèdent qu'une partie des propriétés du nom et n'existent que sous une forme liée à une fonction syntaxique (sujet je ou objet me). On, selon ces critères ferait partie des pronoms non prédicatifs puisque n'existant qu'en position sujet et ne pouvant suivre un présentatif.

On a donc les deux séries :

\begin{tabular}{|l|l|l|l|}
\hline \multirow{2}{*}{ Interlocutoire } & $\begin{array}{l}\text { Pronom ontique } \\
\text { Prédicatif ou disjoint }\end{array}$ & \multicolumn{2}{|c|}{$\begin{array}{c}\text { Plan verbal, Pronom existentiel } \\
\text { Non prédicatif ou conjoint }\end{array}$} \\
\hline & & sujet & objet \\
\hline Non interlocutoire & moi & je & me \\
\cline { 2 - 4 } & toi & tu & te \\
\hline \multirow{2}{*}{ Interlocutoire } & lui, elle & il, elle & le, la, lui \\
\hline Non interlocutoire & nous & on & se, te, vous, nous \\
\cline { 2 - 4 } & vous & nous & nous \\
\hline & eux, elles & vous & vous \\
\hline & $3 P$ indéterminée & ils, elles & les, leur \\
\hline
\end{tabular}

Tableau 3 : Plans nominal et verbal des pronoms personnels

On retiendra dans un premier temps comme traits inhérents de on, les traits /+Humain/, /+Sujet/, /+Pronom/, /-Défini/, /+3S/. En tant que pronom existentiel de la 3S, le on pourrait être assimilé à un pronom anaphorique dont la référence doit être construite, à la différence des pronoms interlocutifs, qui, eux, fonctionnent par référence déictique au contexte énonciatif7. Le fait que ce soit par les pronoms ontiques, (ils correspondent à une dénomination par monstration

\footnotetext{
${ }^{6}$ Je remercie $S$. Bouquet pour les discussions très enrichissantes qui m'ont permis une description systématique des valeurs référentielles du on et de mettre le doigt sur les problèmes de frontières entre les deux linguistiques.

${ }^{7}$ Sauf dans les usages génériques du je, du $t u$, du nous ou du vous.
} 
déictique, ou par topicalisation), que le pronom on peut être glosé dans sa désambiguïsation référentielle tend à conforter cette hypothèse.

Quant à la hiérarchisation des ambiguités à lever selon les pronoms personnels, on pourrait la décrire ainsi: je, $t u<$ nous, vous $<i l$, elle $<$ on. Le on participe à la construction d'une sphère d'empathie à partir du centre qui est le locuteur. Les pronoms nous et vous, généralement classés comme des embrayeurs, construisent une entité multiple qui peut être soit strictement déictique (moi+toi, toi+toi) soit à la fois déictique et anaphorique (moi+lui, toi+lui). Nous et vous supposent donc un travail interprétatif plus complexe que le je et le $t u$. Même si, dans certains genres, le on remplace le nous, la neutralisation du trait /-Défini/ de on implique a priori des opérations plus complexes que celle des pronoms nous ou vous.

\section{Linguistique de la parole et processus interprétatifs}

Une linguistique de la parole doit rendre compte de la donation de la référence par le sujet parlant et des processus interprétatifs (décider de la valeur en langue pertinente en contexte $^{8}$ ) en réception. Le calcul de la référence est le résultat de l'interaction entre traits de l'énonciation et traits de la référenciation.

La référenciation peut être donnée soit par déixis soit par anaphore, soit par les deux à la fois si l'entité construite est multiple.

Dans la référenciation exclusivement déictique, la référence est donnée soit par les pronoms interlocutifs déictiques soit, pour le on, par l'acte de communication en face à face; la référenciation exclusivement déictique peut donc conjoindre pronoms interlocutoires et non interlocutoires avec geste ostensif (arborescences IIa et IIb + DEI). Une linguistique de la référenciation exclusivement déictique suppose la définition de la prosodie et de la gestuelle interlocutoires.

La référenciation interlocutoire non exclusivement déictique conjoint adresse à l'allocutaire et référence anaphorique (IIb-DEI).

Dans la réferenciation anaphorique, la référence de on n'est pas donnée par la coprésence dans la situation énonciative et l'adresse à l'allocutaire mais par un objet du discours présent dans le contexte gauche (anaphore) ou droit (cataphore).

L'énonciation peut être interlocutoire (incluant locuteur et/ou allocutaire) ou non interlocutoire. 1992) :

Dans l'énonciation interlocutoire (+IDX/+ITL), on peut distinguer (Charaudeau,

- l'interlocution allocutive qui met en jeu la déixis, interpelle l'allocutaire avec ses prosodies propres: injonction, impératif, exclamation, interrogation allocutive (par opposition à l'interrogation portant sur des énoncés génériques ou déontiques par exemple), différents modes d'interpellation.

\footnotetext{
${ }^{8}$ Je prends ici un point de vue sémasiologique, qui est le seul possible dans le travail sur corpus. Mais la discussion reste ouverte de savoir si l'étude de la langue en elle-même et pour elle-même n'implique pas de fait une pratique des genres du locuteur grammairien (donc une linguistique de la parole) qui est résumée dans la contextualisation des exemples et la prosodie nécessaire à leur interprétation.
} 
- l'interlocution élocutive: où le locuteur est présent mais non l'allocutaire: la sphère d'empathie est celle du locuteur qui exprime à la $1 \mathrm{~S}$ ses opinions, sentiments, avec des modalités épistémiques, optatives, volitives etc, le on (ou le tu ou le vous) est ici un ego délocuté, qui peut parfois être remplacé par un pronom 3S. Le passage de la $1 \mathrm{~S}$ au on peut se décliner sous différentes formes liées au point de vue: point de vue générique (on $=$ valeur + Hum + Universalité stricte générique), ego en tant que membre du groupe (+Universalité restreinte définie en contexte), ego en tant que membre de la classe (définie en contexte).

- interlocution délocutive: le locuteur et l'allocutaire sont présents en tant que délocutés. L'allocutaire n'est pas interpellé.

Dans l'énonciation non interlocutoire, le discours ne réfère pas aux interlocuteurs et porte sur des objets non interlocutifs.

Dans l'énonciation interlocutoire, la référence du on, locuteur amplifié à l'allocutaire seul (on =nous $=m o i+t o \imath)$ sera inférée de façon différente selon les modalités d'énonciation :

- L'interlocution allocutive va favoriser le basculement de on vers les valeurs interlocutoires (nous ou tu) sans nécessiter la présence de l'embrayeur nous ou des pronoms ontiques moi+toi. Si les pronoms $2 \mathrm{~S}, 2 \mathrm{P}$ ou $1 \mathrm{P}$ ne sont pas complément du verbe, le on est immédiatement identifié au nous (toi+moi) ou au tu. Ex: Pierre à Jacques: "On s'en va? " "Non, on partira après le repas» vs "On t'attend?" où cette interprétation est bloquée par le 2S complément.

- La référence du on (nous $=$ moi+toi) interlocutoire délocutive concerne un discours sur le moi+toi délocuté, ie hors temps de l'énonciation. Les modalités d'énoncé (le plus souvent assertion, description) sont ici plus ambiguës quant à la référence du on et peuvent nécessiter explicitation de la part du locuteur. Ainsi à l'oral, dans l'ex : X dit à $\mathrm{Y}$ : «On s'est trompé((e)s) sur son compte. », l'allocutaire peut interpréter le on soit comme générique, soit comme entité collective n'incluant ni le locuteur ni l'allocutaire, soit comme interlocutif ; l'ambiguïté sera levée soit par l'introduction du nous (qui implique obligatoirement le je) soit par l'introduction des pronoms ontiques. "Toi et moi, on s'est trompé((e)s) sur son compte ». "Nous nous sommes trompé(e)s sur son compte ».

La référenciation interlocutoire non exclusivement déictique conjoint référence interlocutive et référence anaphorique (IIb-DEI). La différenciation entre modalités allocutive et délocutive fonctionne ici aussi.

- La référenciation interlocutoire non exclusivement déictique du locuteur amplifié à un non allocutaire ne peut correspondre qu'à la modalité délocutive : «Moi et Jacques (nous) partons à $5 \mathrm{~h}$ ». Dans cette modalité, le on nous se construit par la présence du je dans le contexte gauche ("J'ai rencontré Jacques hier. On a discuté jusqu'au soir.»); en son absence, le moi référent de on ne peut être élidé qu'avec un circonstanciel : «Pierre, Jacques et moi, on a discuté jusqu'au soir»; «Avec Pierre et Jacques, on a discuté jusqu'au soir ».

«Elle nous aimait, mais elle n'a jamais été tendre, ma mère. Moi aussi, je me méfie de la tendresse. Jamais on ne s'embrassait chez nous, jamais on (=moi+eux) ne se serrait la main, »: Ici, chez nous a été ajouté et jugé nécessaire pour lever l'ambiguité de on. 
On voit que dans l'interlocution allocutive, la valeur nous (moi+toi) de on va être favorisée alors que dans l'interlocution délocutive du locuteur amplifié, le fonctionnement du on s'apparente furieusement à l'anaphore de moittoi ou moitlui et cette valeur de on nécessite soit la présence des pronoms interlocutifs (je ou nous) dans le contexte immédiat soit, dans un contexte autre que le discours direct, l'héritage du je narratorial du récit autobiographique ou homodiégétique.

- Dans le cas de non indexicalité, le on indéfini pur, générique, est massivement présent dans les énoncés doxiques, les énoncés déontiques, gnomiques, de vérité universelle, épistémiques (" on peut penser que»), autonymiques ("comme on les appelle »). Il est lié au présent intemporel de l'énoncé générique.

\section{Les valeurs de on selon les genres}

\subsection{L'analyse fréquentielle de on selon les genres et les $D R$}

Une analyse purement fréquentielle de l'usage du on peut-elle montrer des différences entre les genres définis a priori ?

La valeur comparative choisie pour le taux de fréquence est non pas le $\%$ de on / nombre de mots du genre ou du DR mais le \%o de verbes dont on est le sujet. Il parait en effet plus judicieux de rapporter la fréquence de on à la partie du discours à laquelle il est syntaxiquement lié.

\begin{tabular}{|c|c|c|c|c|c|c|c|}
\cline { 2 - 8 } \multicolumn{1}{c|}{} & \multicolumn{7}{c|}{ ROMANS } \\
\cline { 2 - 9 } \multicolumn{1}{c|}{} & ACN & Barrage & Dix Heur & Moderato & Ravissement & Chartreuse & Total \\
\hline Total & 40,00 & 44,42 & 32,68 & 34,66 & 29,55 & 39,06 & 38,65 \\
\hline DD & 63,00 & 67,15 & 52,85 & 42,19 & 38,97 & 30,59 & 44,41 \\
\hline DI & 23,68 & 18,18 & & & & 65,63 & 32,30 \\
\hline DIN & 49,50 & 0,00 & & & & 80,46 & 43,17 \\
\hline MI & & 0,00 & 39,22 & & & 31,34 & 29,68 \\
\hline Narr & 32,03 & 29,03 & 21,78 & 25,61 & 26,63 & 45,68 & 34,57 \\
\hline Récit1S & & 74,48 & & & 26,09 & & 36,72 \\
\hline MIN & & & 49,53 & & 20,98 & 8,40 & 36,44 \\
\hline
\end{tabular}

Tablean 4 - Le taux \%o de on/nb Verbes dans les romans (discours narratorial et DR)

Note: les cases vides correspondent à des DR de taille insuffisante pour être pris en compte.

\begin{tabular}{|c|c|c|c|c|c|c|c|c|c|c|c|}
\hline & $\begin{array}{c}\text { Discus- } \\
\text { sion }\end{array}$ & \multicolumn{10}{|c|}{ Recueils d'articles } \\
\hline & Parleuse & $\begin{array}{l}\text { Total } \\
\text { article } \\
\text { presse }\end{array}$ & $\begin{array}{c}\text { Entre- } \\
\text { tiens }\end{array}$ & $\begin{array}{c}\text { Propos } \\
\text { recueil- } \\
\text { lis }\end{array}$ & $\begin{array}{l}\text { Récit } \\
\text { hétéro }\end{array}$ & $\begin{array}{l}\text { Récit } \\
\text { auto- } \\
\text { bio }\end{array}$ & $\begin{array}{l}\text { Comm. } \\
\text { Actua }\end{array}$ & $\begin{array}{l}\text { Comm. } \\
\text { CEuvre }\end{array}$ & $\begin{array}{l}\text { Article } \\
\text { criti- } \\
\text { que }\end{array}$ & $\begin{array}{l}\text { Réfle- } \\
\text { xions }\end{array}$ & $\begin{array}{c}\text { Préfa- } \\
\text { ces }\end{array}$ \\
\hline Total & & 83,06 & 80,99 & 71,01 & 86,23 & 82,00 & 82,88 & 89,45 & 77,11 & 96,37 & 76,35 \\
\hline $\mathrm{DD}$ & 71,84 & 77,57 & 86,98 & 54,14 & & & & & & & \\
\hline Narr & & & 57,83 & & & & & & & & \\
\hline
\end{tabular}

Tableau 5 - Le taux \%o de on/nb de Verbes dans les entretiens et articles de presse 


\section{Commentaires des Tableaux $4 \& 5$ :}

Qu'en est-il de l'opposition écrit/oral et de la surreprésentation souvent affirmée de on à l'oral ? On constate d'abord que les taux les plus élevés de on n'appartiennent pas aux genres oraux puisqu'il s'agit des genres « réflexions » $(96,37)$ et «commentaires d'œuvre» $(89,45)$ qui ne comportent pas de DD, suivis immédiatement par les entretiens dissymétriques (87) et les récits hétérodiégétiques (86).

Ensuite que le taux de on du DD dans l'ensemble des articles de presse $(77,5)$ comme celui des entretiens asymétriques (87) est supérieur à celui des entretiens à bâtons rompus : 71,8 (Les Parleuses). Le taux de l'oral représenté dans les romans vient loin derrière $(44,4)$, à une exception près : le récit en face à face à la $1 \mathrm{~S}$ dans Un Barrage $(74,4)$. Ces premiers résultats ne contredisent pas la haute fréquence du on à l'oral, comme l'atteste le fort taux des Parleuses ou des entretiens, mais ils montrent surtout la nécessité de sortir de cette opposition simpliste oral/écrit et de rentrer dans la définition précise des dispositifs énonciatifs des genres.

Qu'en est-il des DD dans les romans? Le taux moyen des DD sur les 6 romans est de 44,4, pour les romans de MD de 52,8. On peut d'abord noter que le taux est ici encore très variable selon les œuvres (de 39 à 67 pour les romans de $\mathrm{MD}$, et seulement de 30,6 pour La Chartrense). De fait La Chartreuse est le seul roman de ce corpus où le taux de on est plus faible dans le DD que dans le discours du narrateur?.

En ce qui concerne le discours du narrateur, le taux de on varie chez Duras de 22 (Dix Heures) à $32\left(L^{\prime} A C N\right)$. Ces différences liées au type de narrateur seront élucidées plus loin. En tout cas, il est clair que le narrateur du roman durassien utilise moins le on que Stendhal dans La Chartreuse $(45,7)$ et moins aussi que le narrateur durassien du récit journalistique (85)

Cette première approche purement fréquentielle montre donc de grosses disparités dans l'usage du on mais aussi des différences entre auteurs, entre narrateurs ou entre DD à l'intérieur d'un genre. De plus, on peut avoir pour des genres différents des taux très proches qui correspondent à des valeurs indexicales complètement divergentes. Cette approche ne permet donc pas de différencier les genres ni évidemment d'interpréter les variations. Pour les interpréter, il faut passer à une analyse grammaticale du morphème.

La démarche adoptée consiste à affecter à chaque occurrence de on du corpus balisé une position dans l'arborescence présentée ci-dessous, en distinguant toujours le discours du narrateur des interlocutions représentées. On décrira la répartition des on sur les différents nœuds de l'arborescence décrite ci-dessous :

\subsection{Les noeuds de l'arborescence représentés dans le corpus sont les suivants ${ }^{10}$}

1.1. Indexicalité extratextuelle interlocutoire du locuteur : $(+\mathrm{IDX} /+\mathrm{EXT} /+\mathrm{ITL} /+\mathrm{LOC} /)$

- non amplifié avec effacement énonciatif :

\footnotetext{
${ }^{9}$ Le discours du narrateur est défini dans un premier temps et première approximation comme le complémentaire des discours rapportés.

${ }^{10}$ Notre numérotation des nœuds ne reprend pas strictement celle de S. Bouquet mais on conserve la logique différentielle.
} 
1.1.1. $-\mathrm{AMP} /+\mathrm{SING} /$ (interlocutoire du locuteur non amplifié) $\mathrm{ON}=\mathrm{Je}$

- amplifié à l'allocutaire exclusivement : (+AMP/+ALLO)

1.1.2. $+\mathrm{SING} /+\mathrm{EXCL} /+\mathrm{MASC}$ vs $-\mathrm{MASC}(\mathrm{ON}=$ nous $=$ moi + toi $)$

1.1.5. $-\mathrm{SING} /+\mathrm{EXCL} /+\mathrm{MASC}$ vs $-\mathrm{MASC}(\mathrm{ON}=$ nous $=$ moi + vous $)$

- amplifié à l'allocutaire et à des personnes hors interlocution (référence déictique amplifiée par anaphore) :

1.2.1.1. +AMP/+ALLO/+SING/-EXCL/+TIERSING/+MASC $(\mathrm{ON}=$ nous $=$ moi+toi+lui)

1.2.1.2. +AMP/+ALLO/+SING/-EXCL/+TIERSING/-MASC

$(\mathrm{ON}=$ nous $=$ moi + toi + elle $)$

1.2.2.1. +AMP/+ALLO/+SING/-EXCL/-TIERSING/+MASC

$(\mathrm{ON}=$ nous $=$ moi + toi + eux $)$

1.2.2.2. +AMP/+ALLO/+SING/-EXCL/-TIERSING/-MASC

$(\mathrm{ON}=$ nous $=$ moi + toi + elles $)$

1.2.3. $(\mathrm{ON}=$ nous $=$ moi + vous $($ vous $=$ toi $+n$ toi $)+$ lui ou elle $)$

1.2.4. $(\mathrm{ON}=$ nous $=$ moi + vous $($ vous $=$ toi $+n$ toi $)+$ eux ou elles $)$

- amplifié par anaphore exclusivement 3S ou 3P :

1.2.5.1. + AMP/-ALLO/+SING/+MASC $(\mathrm{ON}=$ nous $=$ moi + lui $)$

1.2.5.2. $+\mathrm{AMP} /-\mathrm{ALLO} /+\mathrm{SING} /-\mathrm{MASC}(\mathrm{ON}=$ nous $=\mathrm{moi}+$ elle $)$

1.2.6.1. +AMP/-ALLO/-SING/+MASC $(\mathrm{ON}=$ nous $=$ moi + eux $)$

1.2.6.2. +AMP/-ALLO/-SING/-MASC $(\mathrm{ON}=$ nous $=$ moi + elles $)$

1.2. Indexicalité extratextuelle interlocutoire de l'allocutaire :

(+IDX/+EXT/+ITL/-LOC/)

- non amplifié :

1.1.10. $-\mathrm{AMP} /+\mathrm{SING} /+\mathrm{MASC}$ vs $-\mathrm{MASC}(\mathrm{ON}=\mathrm{Tu}$, vous (politesse))

\subsection{Analyse des résultats ${ }^{11}$}

On a réparti dans les deux tableaux 7 et 8 les résultats sur l'indexicalité extratextuelle interlocutoire concernant les textes fictionnels et les textes non fictionnels.

\subsection{1 : Le poids des trois types d'indexicalité}

On constate que l'indexicalité extratextuelle interlocutoire est prépondérante dans les DD de certains romans mais pas dans tous et bien présente dans les récits autobiographiques, et dans le discours narratorial des romans intradiégétiques. Le taux d'indexicalité interlocutoire est nettement plus faible dans les entretiens-discussion (Les Parleuses) que dans les dialogues des romans : il ne s'agit pas du même type d'échange. L'indexicalité intratextuelle est présente dans les récits fictionnels et non fictionnels hétérodiégétiques (le je délocuté du récit homodiégétique basculant dans l'indexicalité extratextuelle), plus faible dans les DD des romans sauf dans La Chartreuse. La non indexicalité (le on d'universalité stricte ou empathique) varie fortement dans les romans selon le type de narrateur; elle est nettement plus fréquente dans les genres non

\footnotetext{
11 Faute de place, je ne discute ici que les résultats sur l'indexicalité interlocutoire et renvoie à la version longue sur le site de MODYCO (http ://www.modyco.fr) pour l'exposé des autres résultats.
} 
fictionnels, réflexions, commentaires d'actualité, entretiens, dans la discussion des Parleuses mais aussi dans les DD de La Chartreuse et à un moindre degré dans le discours narratorial de La Chartreuse.

4.3.2 : l'indexicalité extratextuelle interlocutoire : c'est elle qui est le plus rigidement réglée par le contexte générique, et les résultats montrent que les usages interlocutifs du on sont très restreints par rapport aux descriptions de Moignet.

L'indexicalité extratextuelle interlocutoire du locuteur: Elle est très présente dans certains DD sauf ceux de Moderato et de La Chartreuse, mais aussi dans les récits autobiographiques et à un moindre degré dans les Parleuses et le discours narratorial homodiégétique.

- Du locuteur ou de l'allocutaire non amplifiés: Le remplacement de je ou du tu par on (1.1.1 et 1.1.10) est absent des DD et des entretiens : les usages hypocoristiques ne sont pas représentés dans ce corpus; il n'existe que dans l'allocution narrateur/ narrataire des romans intradiégétiques: on vérifie ici la différence entre narrateur intradiégétique (avec adresse au lecteur: La Chartreuse et $A C N$ ) vs extradiégétique (Moderato, Dix Heures), où ces catégories sont inexistantes. Dans La Chartreuse, le on correspond à l'effacement du narrateur et à l'adresse indirecte au lecteur. Ce on se distingue de celui de l'indexicalité intratextuelle par les temps verbaux : présent et futur de l'interlocution vs temps du récit au passé. Dans le récit intra- et homodiégétique du Ravissement, le narrateur ne peut utiliser le on et s'exprime en je. Dans les articles de presse, l'auteur se désigne plus facilement par je ou par nous (à mettre en rapport avec le fait du non anonymat et du non fictionnel) ; par contre l'adresse au lecteur en on est pratiquée.

- Du locuteur amplifié à l'allocutaire exclusivement $(1.1 .2,1.1 .5)$ : Cette amplification (moi+(toi ou vous)) oppose de façon drastique l'interlocution du DD et celle du rapport narrateur/narrataire de la diégèse enchâssante dans le roman, qui ne l'autorise pas. Elle est absente des articles, sauf de La Vie Matérielle, qui garde la trace de l'interlocution des propos recueillis. Elle peut exister en tant qu'adresse au lecteur dans les articles commentaires d'actualité et commentaires d'œuvres (ex : «On connaît tous des gens qui se situent hors du commun, qui ont la prétention de se connaittre eux-mêmes »); dans les commentaires d'oeuvres, l'auteur prend la place du spectateur ou du lecteur et s'adresse ainsi à lui par un on inclusif. Cette amplification est présente de façon très inégale dans les DD des romans. Sa quasi absence dans les dialogues de La Chartreuse, par opposition aux dialogues du Barrage ou de $l^{\prime} A C N$ peut être mise sur le compte du type de socialité et de registre de langue ou de familiarité, mais sans doute aussi d'une évolution diachronique: elle est présente dans les dialogues à relations familières (Un Barrage, ACN, Dix Heures, Le Ravissement) ; dans le DD de l' $A C N$ on a une forte proportion de cette indexicalité (1.1.2 : toi+moi) lié au poids des dialogues entre l'enfant et l'amant, ainsi que dans le DD du Ravissement. Dans Dix Heures l'amplification à l'allocutaire est au pluriel : il s'agit du multilogue de Maria face aux deux autres personnages. Le degré de familiarité différencie aussi, sur ce type d'amplification, les entretiens asymétriques (Outside, 2 occurrences du fait de l'interviewer et non de l'interviewé) et la discussion à bâtons rompus des Parleuses (48 occurrences). 
Le tableau 6 ci-dessous explicite l'opposition entre amplification du locuteur allocutive et délocutive. Dans l'oral représenté (DD du roman), l'interlocution allocutive s'exprime dans un on sans antécédents, surtout dans les dialogues.

\begin{tabular}{|l|l|r|r|r|r|r|r|r|r|}
\hline +ITL+LOC+AMP & \% temps & PC & PS & IMP & PRES & Fut & Cond & $\begin{array}{l}\text { Nb } \\
\text { tot }\end{array}$ & $\begin{array}{l}\text { Pou- } \\
\text { voir }\end{array}$ \\
\hline +ALLOC/+EXCL & Passé $=12,94$ & 6,97 & 0,00 & 5,97 & 57,21 & 13,93 & 12,44 & 201 & 20,40 \\
\hline +ALLOC/-EXCL & Passé $=6,60$ & 2,83 & 0,00 & 3,77 & 50,00 & 31,13 & 8,49 & 106 & 6,60 \\
\hline -ALLOC & Passé $=56,13$ & 20,05 & 0,00 & 38,08 & 37,03 & 1,42 & 0,94 & 424 & 4,95 \\
\hline
\end{tabular}

Tableau 6 - Temps morphologique et indexicalité interlocutoire du locuteur

(à droite :\% de on sujet de pouvoir)

L'amplification allocutive est corrélée à des emplois préférentiels du présent, impératif, ventif aller $+v b$, futur, conditionnel de suggestion (invite ou imaginaire). Ces traits sont partagés par l'amplification à l'allocutaire et à des tiers. Dans l'amplification du locuteur à un tiers exclusivement, interlocution délocutive, le on est introduit par un SN parataxique et le nous qui l'accompagne (" nous sommes des petits enfants maigres, mon frère et moi. On est battus ensemble »), ou par un SP («On se voit encore assez souvent avec cet ami »); la répartition des temps s'inverse.

Amplification du locuteur exclusivement par anaphore (1.2.5 et 1.2.6: moi+lui ou moi + eux $)$ : cette catégorie peut recouvrir deux types de discours : i) d'un côté le récit sur soi, bien représenté dans le discours du narrateur homodiégétique (19\% et absent dans l'hétérodiégétique), dans le récit évènementiel à la $1 \mathrm{~S}$ du Barrage (77\%), dans les récits évènementiels journalistiques autobiographiques (33,12\% dans la Vie Matérielle, 57,5\% dans Outside, 34\% dans Le Monde extérieur); dans ce cas, les occurrences de on sont toujours précédées du je narratorial ou du nous. Les temps verbaux sont ceux du récit (PC, imparfait, présent de narration, absence du PS) ; ii) d'autre part le discours du sujet en tant que membre du groupe (moi+eux) souvent opposé à un autre groupe : ce dernier est présent dans le DD de certains romans où la configuration nous/eux (moi et ma famille face à l'environnement hostile) constitue une trame centrale (roman de formation du Barrage); dans les entretiens d'Outside, les réflexions de la Vie Matérielle, l'amplification anaphorique plurielle du locuteur, ne relève pas du récit, traduit la description du vécu en tant que membre du groupe (hommes/femmes, immigrés). Ici c'est le présent d'habitude, qui domine.

On voit là que le mode d'amplification allié à la sémantique des temps verbaux doit permettre de différencier des types d'énoncés signes de types de discours.

\section{Conclusion}

Quant à la méthode, le travail commun avec S. Bouquet a montré la complémentarité des deux démarches : systématicité de la description grammaticale, mais aussi apports de la confrontation aux textes pour l'enrichissement de cette description ${ }^{12}$. Quant aux résultats, la démarche de croisement des traits grammaticaux

12 L'affectation d'une occurrence à un nœud de l'arborescence (catégorisation) obligeant le locuteur grammairien à une observation différentielle des critères portés par le contexte, le travail sur corpus 
de on et des traits de genres s'avère particulièrement fructueuse pour ce qui est de l'indexicalité interlocutoire, dans la mesure où le genre définit des modalités précises de l'interlocution.

Hjelmslev disait que l'on ne peut «conclure inductivement de l'usage à la norme; la latitude de variabilité est toujours moins grande dans l'usage que dans la norme et un usage donné ne constitue qu'une réalisation de certaines possibilités admises par la norme sans les épuiser ». Cela se vérifie dans notre cas, puisque les occurrences de on dans notre corpus sont loin de représenter l'ensemble de l'arborescence définie par S. Bouquet. On peut noter aussi que les genres seconds complexifient notablement la normativité interlocutive.

Concernant la notion de genre, ce travail montre que la valeur locale du morphème dépend des propriétés interlocutoires du texte dans son entier (on a vu comment le type de narrateur contraint les valeurs interlocutoires possibles du on) comme des propriétés interlocutoires plus locales des séquences textuelles comme des normes de socialité de l'univers représenté. Concernant les normes de socialité (cf. l'opposition interview vs discussion à bâtons rompus), les variations diachroniques à l'intérieur d'un genre en sont un indice : disparition de l'indexicalité interlocutoire de l'allocutaire en on dans le DD du roman contemporain, encore présente dans le DD de La Chartreuse. L'absence du on interlocutif (moittoi ou vous) dans le DD de La Chartreuse, fort présent dans le DD des romans durassiens met en jeu des normes de socialité différentes.

Concernant l'oral, nous avons mis en évidence des répartitions très contrastées selon les genres oraux ; à l'intérieur de l'oral on est amené à distinguer les modalités de l'énonciation interlocutoire (interlocution allocutive vs interlocution délocutive). Il faut donc descendre bien en deçà de l'étiquette générique du texte global et de la séquence textuelle (DD...) et des "modalités d'énonciation » traditionnellement reconnues ${ }^{13}$. Il est probable que l'analyse topologique des valeurs de on, rafales, densité, enchâ̂nements mettrait sur la voie d'une reconnaissance des types d'énoncés caractéristiques des types de discours à l'intérieur d'un même texte. Dans la lecture d'un article de presse par exemple, la construction du sous-genre non déclaré récit autobiographique se fait progressivement à l'intérieur d'un genre déjà donné, grâce à la récurrence temps/ personne, et l'héritage du je autobiographique favorisera l'affectation à on de la valeur +LOC/+AMP/-ALLOC, ce qui n'aura pas lieu dans un texte de réflexion générale ou de commentaire d'actualité.

Cette analyse du on devra donc être complétée selon plusieurs axes : Il serait utile de mener l'analyse sur corpus du on à l'intérieur du paradigme complet des pronoms personnels et non isolément, comme d'analyser les formes vicariantes du on non sujet. Cela permettrait d'explorer les rapports entre choix de la référence en on et

reconfigure, de façon plus riche que la connaissance métalinguistique du locuteur natif, l'élaboration d'hypothèses sur les corrélats suprasegmentaux des phénomènes grammaticaux et syntaxiques, dans la mesure où le sens du texte dans ses contraintes pragmatiques doit rester en ligne de mire. Cette démarche permet au linguiste de concevoir, à partir de l'observation du corpus des expérimentations d'exemples jouant sur des contraintes de portée plus ou moins locale.

${ }^{13}$ La notion de «modalité d'énonciation » ou «types de phrases » restant tout à fait insuffisante pour caractériser l'existence et le type d'une interlocution. 
actantiation. La suite de l'analyse doit consister à préciser la relation entre les traits suprasegmentaux décrits et les propriétés syntaxiques de l'énoncé : types d'arguments et temps et types des verbes dont on est le sujet ou argument autre, étant entendu que la valeur sémantique des temps est elle-même fonction des traits suprasegmentaux. Grâce à une analyse syntaxique et lexicale précise des énoncés qualifiés par leurs traits suprasegmentaux on pourra élaborer des grammaires locales du on, qui permettraient d'articuler types d'énoncés, types de discours et traits de genres.

\section{RÉFÉRENCES BIBLIOGRAPHIQUES}

Adam, J.-M. \& Heidman, U. (2006) «Six propositions pour l'étude de la généricité », in R. Baroni \& M. Macé (eds) : Le savoir des genres, La Licorne, PUR, vol. 79, pp. 21-34.

ATLANi F. (1984). «ON l'illusioniste » in La langue au ras du texte, 13-29. Presses universitaires de Lille.

BAKHTine, M., 1984 [1952-53], Esthétique de la création verbale, Paris, Gallimard.

Benveniste, E. (1966). «La nature des pronoms », in Problèmes de linguistique générale I, 251-257, Paris, Gallimard.

BLANCHE-BenVEniste, C. (1990). Le français parlé : études grammaticales, Paris, CNRS.

BOUQUET, S., 2004. «Sémiotique grammaticale et sémantique des (genres de) jeux de langage: les pronoms personnels clitiques en français », Langages, 153, pp. 28-40.

FLUDERNICK, M. (1996). «Towards a « Natural » Narratology », Routledge, London, New-York.

FOREST, R., (2003). «Empathie linguistique et point de vue », Cabiers de praxématique, n 41, 85-104.

FOREST, R. (2003) Critique de la raison linguistique, Paris, L'Harmattan.

FrANÇOIS, J. (1984). "Analyse énonciative des équivalents allemands du pronom indéfini on », in G. Kleiber (éd), Recherches linguistiques, n X, 37-73, université de Metz/Klincksieck.

GenetTe, G. (1983). Nouveau discours du récit, Paris, Seuil.

JaYez, J. \& TovenA, L. (2006), "Indéfinis et identification», in F. Corblin, S. Ferrando, L. Kupferman (dir.) Indéfini et prédication, 67-80, Paris, PUPS.

Joly, A. (1987). Essais de systématique énonciative, PUL.

LEEMAN, D. (1991). « ON, thème », Linguisticae Informationes, XV/1, 101-113.

LEEMAN, D. (2002). «Je me moi, allomorphes ou différentes facettes de la première personne? « in D. Lagorgette et M. Lignereux, Comme la lettre dit la vie, LINX, $\mathrm{n}^{\circ}$ spécial

Maingueneau, D. (2000). «Instances frontières et angélisme narratif », Langue française, 128 ; pp. 74-95.

MALRIEU, D. (1997). "Reconnaissance de catégories discursives et repérages énonciatifs: l'exemple des hypothèses dans les textes scientifiques », Revue de Sémantique et Pragmatique, $\mathrm{n}^{\circ} 1$, pp. 147-163.

Malrieu, D. \& Rastier, F. (2001). «Genres et variations morphosyntaxiques », T.A.L., vol. 42, $\mathrm{n}^{\circ} 2,547-577$. 
MALRiEU, D. 2004. Linguistique de corpus, genres textuels, temps et personnes, Langages, $\mathrm{n}^{\circ} 153,73-85$.

MALRIEU, D. (2007) : "Quel balisage du roman contemporain ?», in Actes des Journées d'études internationales "Corpora et questionnements du littéraire », Paris X-Nanterre, 15 \& 16 novembre 2005, nº spécial de LINX 2007.

MOIGNET, G. (1965). Le pronom personnel français : essai de psycho-systématique historique, Klincksieck.

Muller, C. (1979). "Sur les emplois personnels du on, in Langue française et linguistique quantitative, Slatkine, p. 65-72.

RABATEL, A. (2001). "La valeur de «on» pronom indéfini/pronom personnel dans les perceptions représentées. "L'Information grammaticale, $\mathrm{n}^{\circ} 88$.

RAstier, F. (2001). «Arts et sciences du texte «PUF.

SCHAPIRA, C. (2006). «On pronom indéfini », in F. Corblin, S. Ferrando \& L. Kupferman (dir.) Indéfini et prédication, PUPS, 507-518.

SCHAEFFER, J.M. (2006). «Des genres discursifs aux genres littéraires : quelles catégorisations pour quels faits textuels ? «in R. Baroni \& M. Macé (eds): Le savoir des genres, La Licorne, PUR, vol. 79.

TAmba I. (1989). "De la double énigme de on au concept de pronom et de personne linguistique en français et en japonais », Sophia Linguistica, 27, 5-23. 


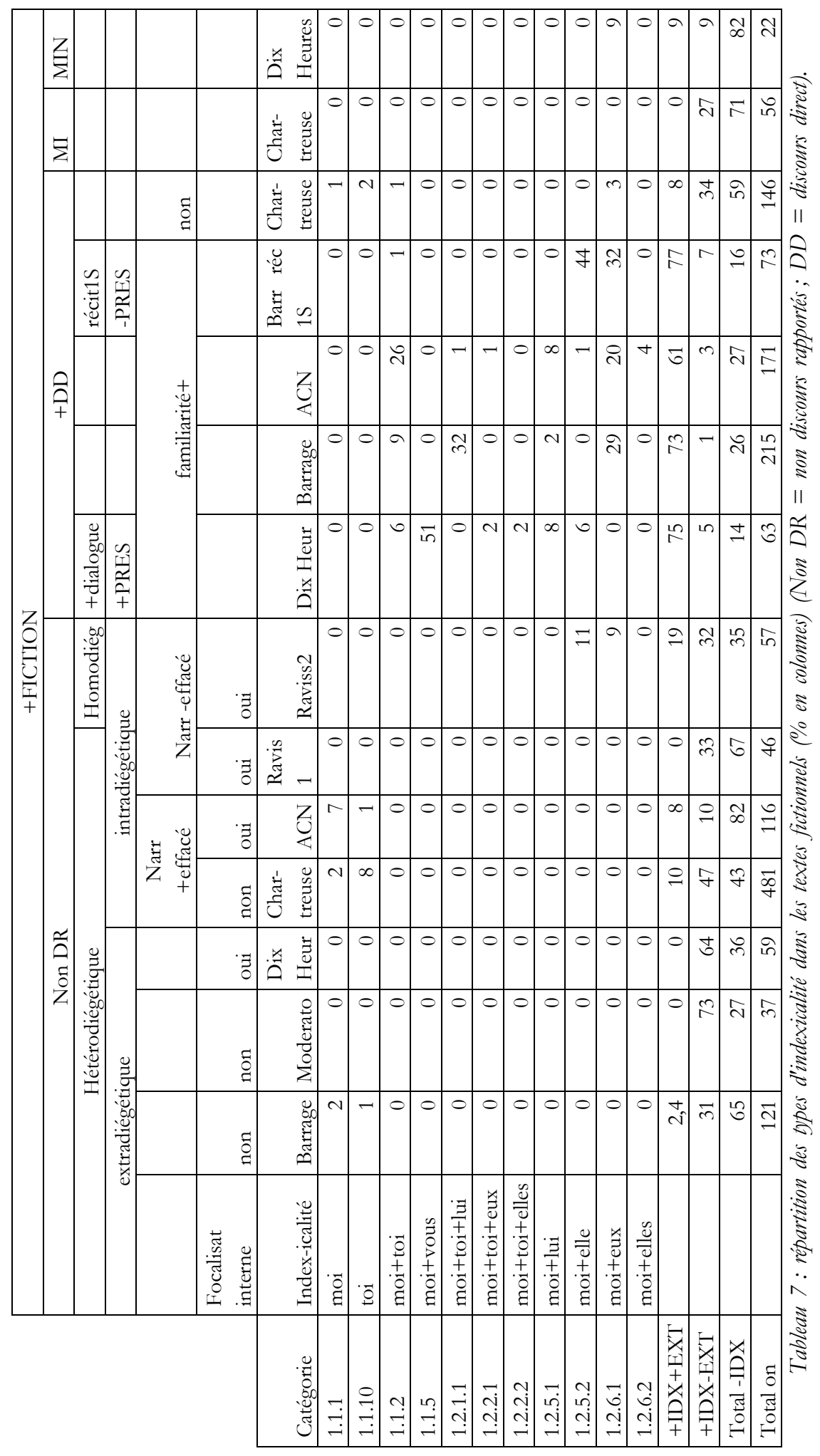




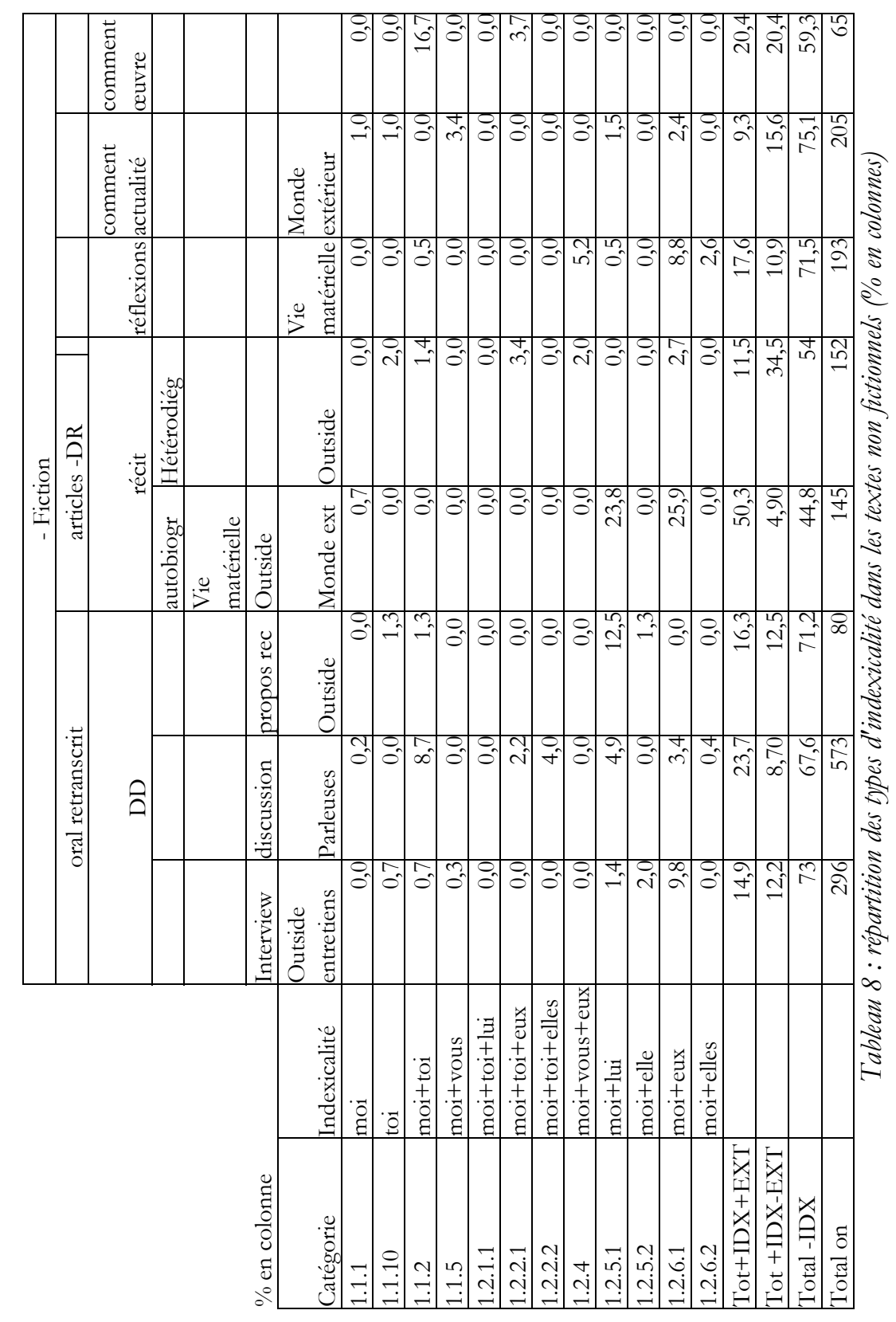

